\title{
An Effective Approach of Noise Analysis on Images
}

\author{
Gourav Kumar Javeriya \\ Govt. Engineering College, \\ Ajmer, Rajasthan
}

\author{
Deepak Gupta \\ Asst. Professor, Department of \\ CS \\ Govt. Engineering College, \\ Ajmer,Rajasthan
}

\author{
Anil Kumar Dahiya \\ Professor, Senior Member \\ IEEE \\ Manipal University, \\ Jaipur,Rajasthan
}

\begin{abstract}
Here it is represented an image analysis technique using both noising \& de-noising process. By taking a simple image of different formats we added a noise i.e. Gaussian noise to the particular image and then the calculation of SNR(SIGNALTO-NOISE RATIO) and PSNR(PEAK SIGNAL-TO-NOISE RATIO) is performed based on the image formats such as jpeg, png, etc. Although there are various types of noise but we considered here the Gaussian noise only. The calculation done is also according to the different functions (blocking, nlfilter, etc.) and the related categories applied on the simple images. After then the image de-noising is performed on the obtained noised image and the image without any applied function in order to check the comparative analysis of the image containing noise and the de-noised image. The image observed is not as an exact replica of the previous image without noise. Also, there is a vast difference between the SNR and PSNR values of the noised and de-noised image. An image transferred by the sender, if get some distortion like Gaussian noise, then after de-noising process the image observed on the receiver side may be not real and exact, but have less distortion which is a great advantage.
\end{abstract}

\section{General Terms}

Noise Analysis, Comparison of image properties like psnr and snr, comparative analysis of different image formats.

\section{Keywords}

Blocking Function, Gaussian Noise, Image, Peak Signal-toNoise Ratio, Signal-to-Noise Ratio.

\section{INTRODUCTION}

Image Noise is random(not present in the object imaged) variation of brightness or color information in images, and is usually an aspect of electric noise. It can be produced by the sensor and circuitry of a scanner or digital camera. Image noise can also originate in film grain and in the unavoidable shot noise of an ideal photon detector. Image noise is an undesirable by-product of image capture that spurious and extraneous information. The original meaning of "noise" was and remains unwanted signal: unwanted electrical fluctuations in signals by $\mathrm{AM}$ radios caused audible acoustic noise ("static"). By analogy unwanted electrical fluctuations themselves came to be known as "noise". Image noise is, of course, inaudible. The magnitude of image noise can range from almost imperceptible specks on a digital photograph taken in good light, to optical and radio-astronomical images that are almost entirely noise, from which a small amount of information can be derived by sophisticated processing(a noise level that would be totally unacceptable in a photograph since it would be impossible to determine even what the subject was). Also, there are various categories of noise. Principle sources of Gaussian noise in images arise during acquisition e.g. sensor noise caused by poor illumination and/or high temperature, and or transmission e.g. electronic circuit noise. The standard model of this noise is additive, independent at each pixel and independent of the signal intensity, caused primarily by thermal noise, including that which comes from the reset noise of capacitors. Another noise is Impulsive noise which is sometimes called as salt-andpepper noise or spike noise. An image containing salt-andpepper noise will have dark pixels in bright regions and bright pixels in dark regions. The next is the shot noise, which is the dominant noise in the lighter parts of an image from an image sensor is typically that caused by statistical quantum fluctuations, that is, variation in the number of photons sensed at a given exposure level. Also, the Quantization Noise i.e. uniform noise is the noise caused by quantizing the pixels of a sensed image to a number of discrete levels is known as quantization noise. Some noise sources show up with a significant orientation of images. Such noise is called as Anisotropic Noise. The last one is the Film grain, which is usually regarded as a nearly isotropic (non-oriented) noise source. Its effect is made worse by the distribution of silver halide grains in the film also being random.

\section{PRINCIPLE OF NOISING AND DE- NOISING}

It is a concept of image processing given by Gonzalez and Woods [1].The principle is to add noise to an image and to calculate the PSNR and SNR values of the noised image. These values are calculated on different types of images. Although, there are various categories of noise present and discussed in this paper, but, we can use any type of noise depending on our choice. Here, in this paper we have taken Gaussian Noise in order to calculate the required values. Gaussian noise is added to an image.

Consider the figure 1 in which we have taken a simple image and figure 2 in which Gaussian noise is added to it. Here, in the figure 2, the noisy image has white colored dots in each and every pixels because the standard model of this noise is additive, independent at each pixel and independent of the signal intensity, caused primarily by thermal noise.

Now, De-noising of a noisy image obtained, is performed. Calculation of the PSNR and SNR values of the de-noised image is done in order to do comparative analysis of the values obtained with noise and image obtained after de-noising. An excellent de-noising concept is given by A. Buades and S.B. Chang [2][3]. Again, consider figure 2 in which the Gaussian Noise is added to an image and figure 3 in which the de-noising of an obtained noisy image is done. 


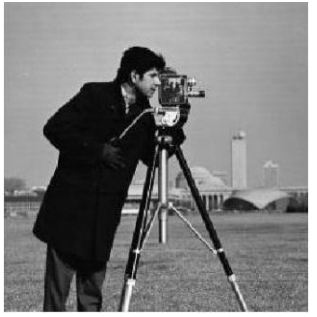

Fig.1. Simple image

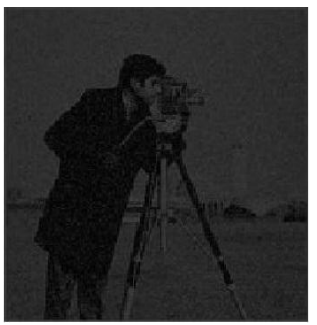

Fig.3. De-Noised Image

Here, in the above figure 3, the de-noised image shown is not represented in the form of an exact replica of the real image.It is having some deformities which is discussed in this paper.To analyze further descriptive statistics of an image, various categories of functions are applied on both the noised and denoised image.Now, the next step is the calculation of PSNR and SNR values of both the noised image. After that the image deniosing is done in order to observe the quality of an image. As per calculations obtained, there is a vast difference in the PSNR and SNR values obtained for the simple image(which is the original image withoutany added noise and function) and for the de-noised image. Also, the quality of an image observed after de-noising was not an exact as compared to the simple image. Another thing which must be kept in mind is that the dimensions of all the images must be same for the calculations of PSNR and SNR values.

\section{PROPOSED WORK}

Here, in the proposed work we have added Gaussian noise to varoius types of image formats. After adding noise, we have calculated the PSNR and SNR values of that noisy images. In the next step we have added varoius functions to the noised images and again calculated the PSNR and SNR values. Final step is the removal of added noise and then again the calculation of the values was done. This made the comparative analysis of various types of images based on noised images, function added images and de-noised images. There are various functions but for the simplicity we have shown the blocking functiononly in this paper. It is cleary explained by T.S.Huang[4]. Consider the figure below showing the noising and de-noising of an image:

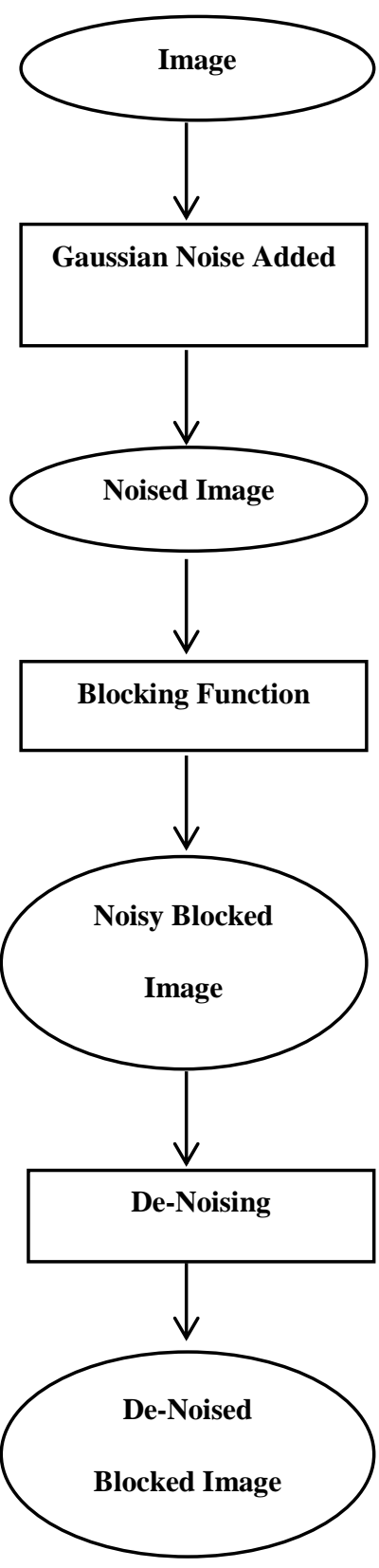

Fig. 4. Noising and De-noising with applied blocking function

\subsection{Effect of gaussian noise on various image formats.}

The calculation is based on P.J. Ready theory [5]. D. S. Turaga also proposed PSNR estimation [6]. Gaussian Noise is added to various image formats for the SNR values and the representation is as follows:

TABLE 1. SNR values of different image formats

\begin{tabular}{|c|c|c|c|c|c|}
\hline $\begin{array}{c}\text { VALUE } \\
\text { IMAGES }\end{array}$ & JPEG & BMP & PNG & GIF & TIFF \\
\hline \multirow{2}{*}{ SNR } & 200. & 242. & 203. & 214. & 198. \\
& 9385 & 9319 & 0065 & 3502 & 0954 \\
\hline
\end{tabular}




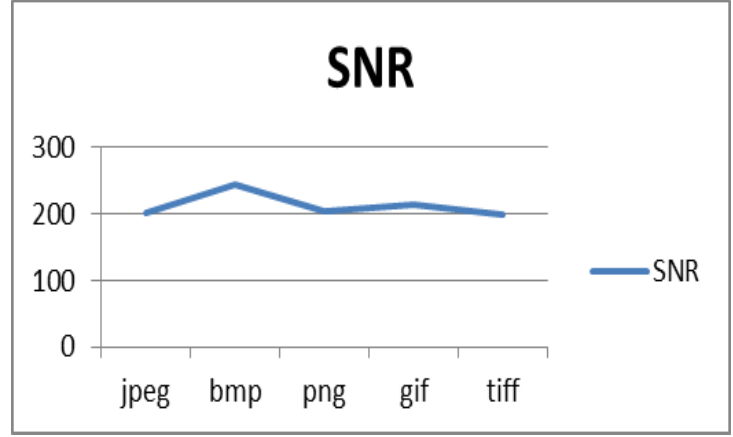

Fig. 5. SNR Graph

\subsection{Blocking effect on noised(gaussian) image.}

Blocking function divides the image into blocks. Therefore, the blocking of a noisy image is performed and the presentation is as follows:

TABLE 2. SNR with Blocking function values of different image formats

\begin{tabular}{|c|c|c|c|c|c|}
\hline $\begin{array}{c}\text { FUNCTIONS/ } \\
\text { IMAGES }\end{array}$ & JPEG & BMP & PNG & GIF & TIFF \\
\hline dct & 205. & 200. & 235. & 152. & 222. \\
& 8156 & 8372 & 1334 & 1132 & 3122 \\
\hline Std & 251. & 211. & 254. & 160. & 253. \\
& 3406 & 2933 & 5732 & 0854 & 6032 \\
\hline inline & 251. & 211. & 222. & 160. & 253. \\
& 4170 & 4049 & 3121 & 6146 & 6040 \\
\hline mean & 202. & 231. & 222. & 148. & 203. \\
& 6681 & 5706 & 0391 & 3010 & 2777 \\
\hline
\end{tabular}

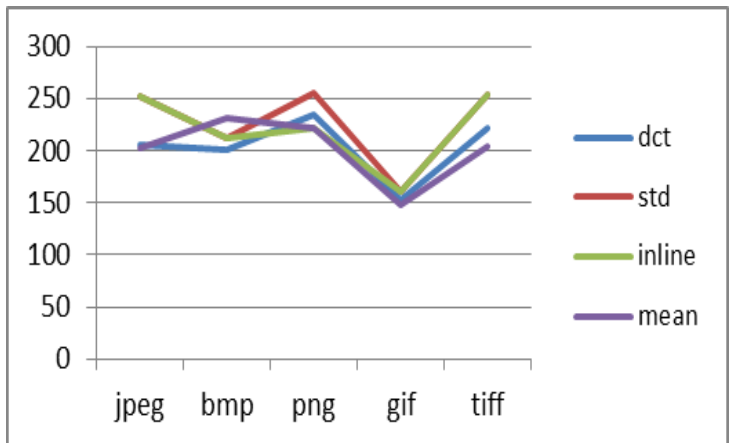

Fig. 6. SNR with blocking function Graph

\subsection{De-noising the noised image.}

The noised image is de-noised for the SNR values and presentation is as follows:

TABLE 3. SNR values of de-noised image formats

\begin{tabular}{|c|c|c|c|c|c|}
\hline $\begin{array}{c}\text { VALUE\ } \\
\text { IMAGES }\end{array}$ & JPEG & BMP & PNG & GIF & TIFF \\
\hline \multirow{2}{*}{ SNR } & 177. & 170. & 172. & 209. & 139. \\
& 7442 & 6795 & 3145 & 9615 & 0670 \\
& & & & & \\
\hline
\end{tabular}

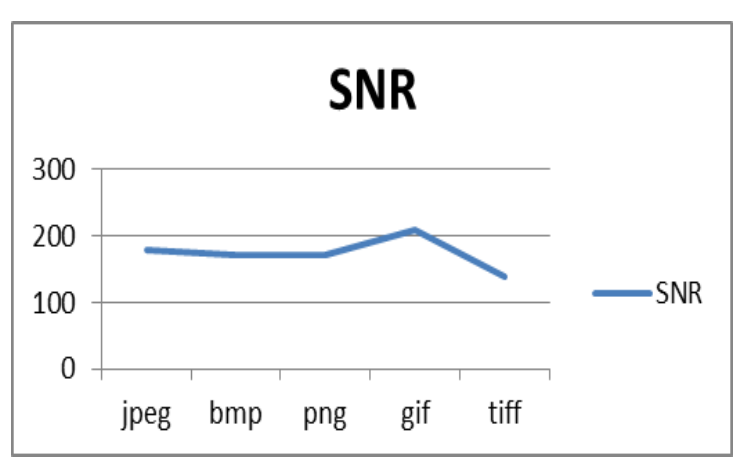

Fig. 7. SNR de-noised image Graph

\subsection{De-noising the noisy blocked image.}

The noisy blocked image is de-noised for SNR values and the presentation is as follows:

TABLE 4. SNR with Blocking function values of denoised image formats

\begin{tabular}{|c|c|c|c|c|c|}
\hline $\begin{array}{c}\text { FUNCTIONS/ } \\
\text { IMAGES }\end{array}$ & JPEG & BMP & PNG & GIF & TIFF \\
\hline dct & 115. & 236. & 99. & 110. & 113. \\
& 3467 & 7438 & 7786 & 9409 & 7365 \\
\hline std & 84. & 153. & 183. & 96. & 175. \\
& 9414 & 3467 & 3529 & 5611 & 5630 \\
\hline inline & 84. & 126. & 224. & 96. & 199. \\
& 9470 & 8365 & 8107 & 5551 & 4001 \\
\hline mean & 100. & 112. & 101. & 147. & 189. \\
& 8533 & 4702 & 4572 & 8883 & 0286 \\
\hline
\end{tabular}

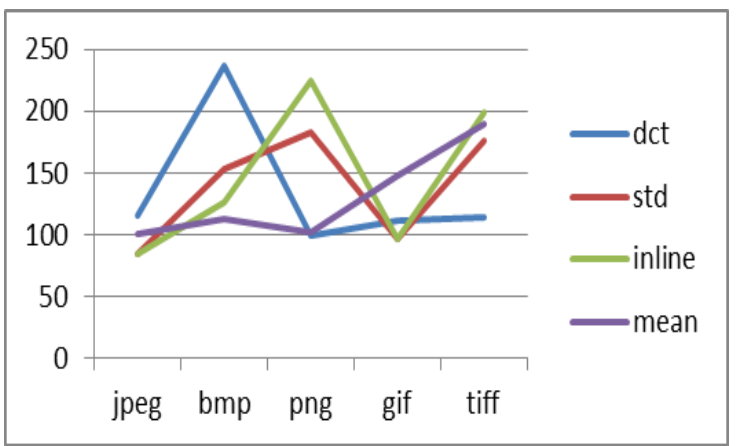

Fig. 8. SNR with blocking function of de-noised image

$$
\text { Graph }
$$

\subsection{Effect og gaussian noise on various image formats for psnr}

Linfeng Guo proposed an algorithm for PSNR estimation [7]. Same is the case here, Gaussian Noise is added to various image formats for the PSNR values and the representation is as follows: 
TABLE 5. PSNR values of different image formats

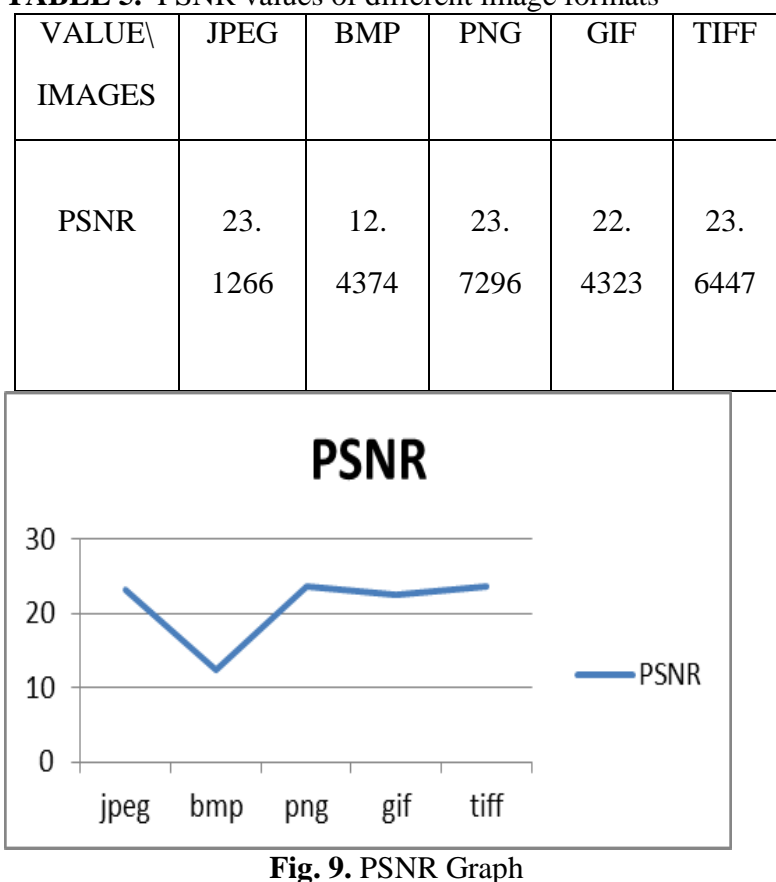

\subsection{Blocking effect on noised(gaussian) image.}

Blocking function divides the image into blocks. Therefore, the blocking of a noisy image is performed and the presentation is as follows:

TABLE 6. PSNR with Blocking function values of different image formats

\begin{tabular}{|c|c|c|c|c|c|}
\hline $\begin{array}{c}\text { FUNCTIONS/ } \\
\text { IMAGES }\end{array}$ & JPEG & BMP & PNG & GIF & TIFF \\
\hline \multirow{2}{*}{ dct } & 7. & 6. & 9. & 7. & 25. \\
& 6782 & 1387 & 7671 & 6829 & 2341 \\
\hline \multirow{2}{*}{ std } & 21. & 5. & 24. & 11. & 25. \\
& 9755 & 4833 & 9972 & 3029 & 4261 \\
\hline \multirow{2}{*}{ inline } & 21. & 5. & 5. & 11. & 25. \\
& 9799 & 4887 & 7088 & 3122 & 4104 \\
\hline \multirow{2}{*}{ mean } & 23. & 11. & 23. & 23. & 23. \\
& 2483 & 9171 & 8939 & 8842 & 7505 \\
\hline
\end{tabular}

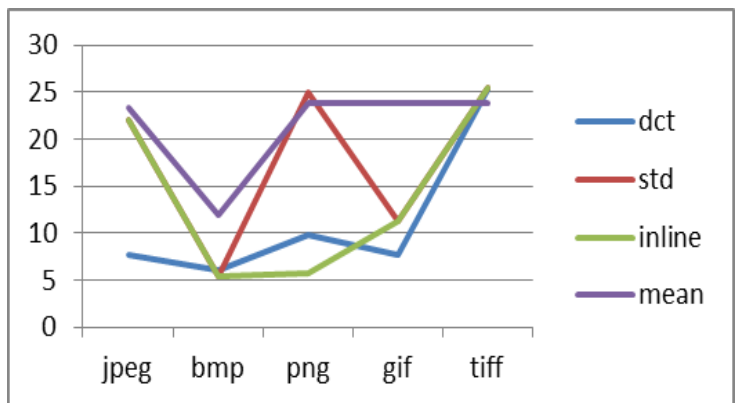

Fig. 10. PSNR with blocking function Graph

\subsection{De-noising the noised image.}

The noised image is de-noised for the PSNR values and presentation is as follows:

TABLE 7. SNR values of de-noised image formats

\begin{tabular}{|c|c|c|c|c|c|}
\hline VALUEI & JPEG & BMP & PNG & GIF & TIFF \\
\hline IMAGES & & & & & \\
\hline & & & & & \\
& 5309 & 5675 & 5441 & 2341 & 3033 \\
& & & & & \\
\hline
\end{tabular}

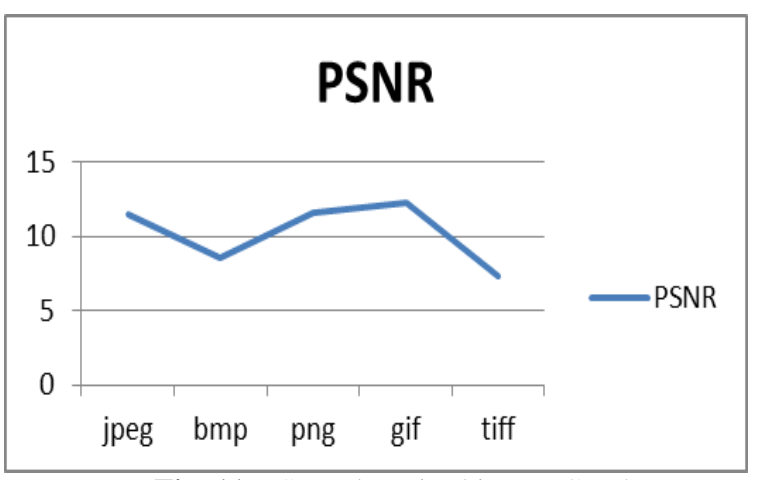

Fig. 11. PSNR de-noised image Graph

\subsection{De-noising the noisy blocked image.}

The noisy blocked image is de-noised for PSNR values and the presentation is as follows:

TABLE 8. PSNR with Blocking function values of denoised image formats

\begin{tabular}{|c|c|c|c|c|c|}
\hline $\begin{array}{l}\text { FUNCTIONS/ } \\
\text { IMAGES }\end{array}$ & JPEG & BMP & PNG & GIF & TIFF \\
\hline dct & $\begin{array}{c}6 . \\
3731\end{array}$ & $\begin{array}{c}8 . \\
7589\end{array}$ & $\begin{array}{c}5 . \\
0719\end{array}$ & $\begin{array}{c}6 . \\
3891\end{array}$ & $\begin{array}{c}5 . \\
9814\end{array}$ \\
\hline std & $\begin{array}{c}4 . \\
6700\end{array}$ & $\begin{array}{c}6 . \\
8499\end{array}$ & $\begin{array}{c}8 . \\
1739\end{array}$ & $\begin{array}{c}5 . \\
6117\end{array}$ & $\begin{array}{c}7 . \\
7605\end{array}$ \\
\hline inline & $\begin{array}{c}4 . \\
6648\end{array}$ & $\begin{array}{c}4 . \\
8464\end{array}$ & $\begin{array}{c}7 . \\
5224\end{array}$ & $\begin{array}{c}5 . \\
6112\end{array}$ & $\begin{array}{c}9 . \\
5099\end{array}$ \\
\hline mean & $\begin{array}{c}7 . \\
6621\end{array}$ & $\begin{array}{c}5 . \\
8414\end{array}$ & $\begin{array}{c}6 . \\
2244\end{array}$ & $\begin{array}{c}7 . \\
9001\end{array}$ & $\begin{array}{c}13 . \\
3240\end{array}$ \\
\hline
\end{tabular}

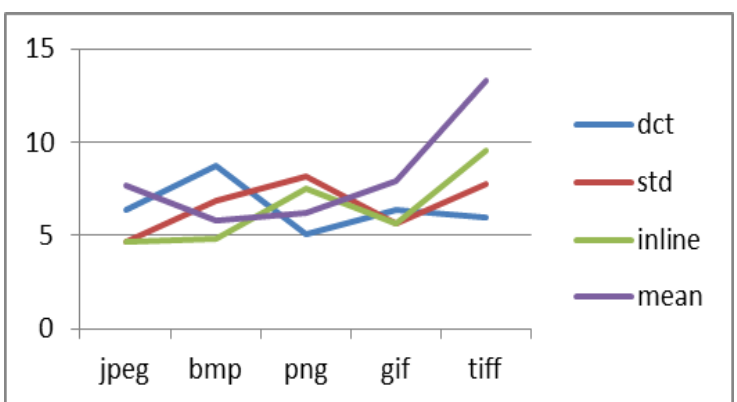

Fig. 12. PSNR with blocking function of de-noised image Graph 


\section{CONCLUSION}

As per the noising and de-noising algorithm applied on various image formats, there were some of the results and conclusions which were analyzed during the proposed work. After calculations of PSNR and SNR values of the noised and de-noised image there was a vast difference between the observations. The main reason was due to the addition of noise which created a difference. But, after de-noising the noised image and calculating the required values, again a vast difference was observed. Also, the quality of an image after removing noise was not an exact replica of the real image. Finally, the most important thing observed was the dimensions, if an image must be same in order to observe exact value, else it may give garbage value.

As per the calculations we conclude that an image transferred by the sender, if get some distortion like Gaussian noise, then after de-noising process the image observed on the receiver side may be not real and exact, but have less distortion which is one of the requirement and also a great advantage.

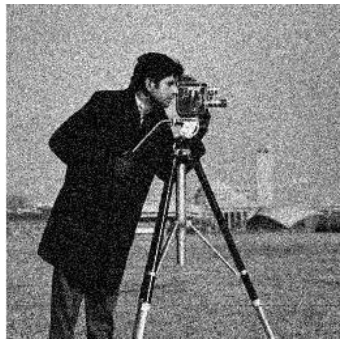

Fig. 13.Adding Gaussian Noise to Fig. 1. $(\mathrm{SNR}=200.9385$, $\mathrm{PSNR}=23.1266$

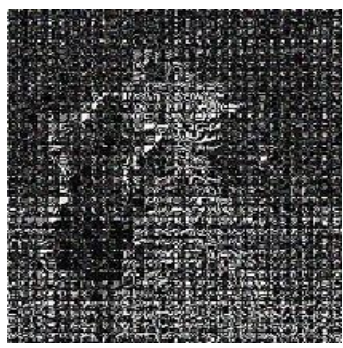

Fig. 15.Blocking function applied on Fig. 13 with det value, $(\mathrm{SNR}=205.8156$, $\mathrm{PSNR}=7.6782$

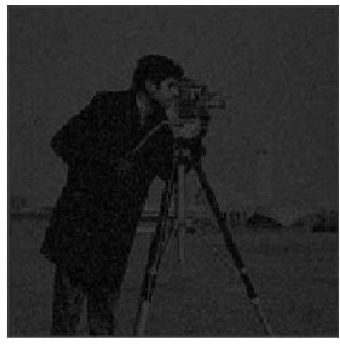

Fig. 14. Removing Gaussian Noise from Fig. 13 $(\mathrm{SNR}=170.7442$, $\mathrm{PSNR}=11.5309)$

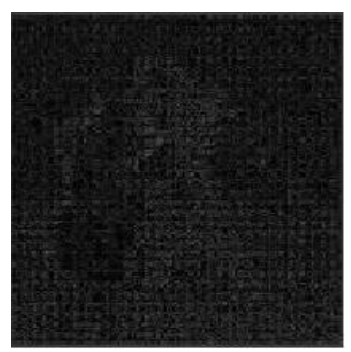

Fig. 16. De-noising Fig. 14. image $(\mathrm{SNR}=115.3467$, $\mathrm{PSNR}=6.3731$ )

\section{ACKNOWLEDGMENTS}

Through this page, I express my heartfelt thanks to Mr. Deepaka Gupta, Assistant Professor, Government Engineering College Ajmer, who gave me the opportunity to work on this topic and inspired me to carry forward this work as a challenge.

I especially thank to Mr. Dhirendra Mathur, (Head, Department of Computer Engineering \& Information Technology, Government Engineering College, Ajmer), Mrs. Prakriti Trivedi, and Dr. Rakesh Rathi (Assistant Professor, Department of Computer Engineering \& Information Technology, Government Engineering College, Ajmer) for their valuable guidance.

I am grateful to all the faculty members and non teaching staff of the Dept. Computer Engineering \& Information Technology department at Government Engineering College, Ajmer.

\section{REFERENCES}

[1] Gonzalez and Woods, Digital Image Processing, $2^{\text {nd }}$ edition, Prentice Hall, 2002. Sec.8.5.2.

[2] A. Buades, B.Coll, and J.M. Morel, A review of image denoising algorithms, with a new one, Multiscale Modeling and Simulation, 4(2): 490-530, 2005.

[3] S.G. Chang, B. Yu, and M.Vetterli, Adaptive wavelet thresholding for image denoising and compression, IEEE Trans. on Image Processing, 9(9): 1532-1546, 2000.

[4] M. Gharavi-Alkhansari and T.S. Huang, A Fractal-Based Image Block-Coding Algorithm, Proceedings ICASSP 93, V pp. 345-348.

[5] P. J. Ready and P. A. Wintz, "Information ectraction, SNR improvement, and data compression in multispectal imagery" IEEE Trans. Commun., vol. 21, pp. 1123-1130, 1973.

[6] D.S. Turaga, C. Yingwei and J. Caviedes, "No reference SNR estimation for compressed pictures,"Proc. IEEE International Conference on Image Processing, vol.3, pp.61-64, June 2002

[7] PSNR- BASED OPTIMIZATION OF JPEG-BASELINE COMPRESSION ON COLOR IMAGES, Linfeng Guo, BrainMedia LLC, New York, New York 10016, USA

[8]http://www.math.ust.hk/ masyleung/Teaching/CAS/MAT LAB/image/images/cameraman.jpg 